M. OZAWA

KŌDAI MATH. SEM. REP.

27 (1976), 373-378

\title{
SUFFICIENT CONDITIONS FOR AN ENTIRE FUNCTION TO BE PSEUDO-PRIME
}

\author{
By Mitsuru Ozawa
}

\section{§1. Introduction.}

A meromorphic function $F(z)=f(g(z))$ is said to have $f(z)$ and $g(z)$ as left and right factors respectively, provided that $f$ is meromorphic and $g$ is entire ( $g$ may be meromorphic when $f$ is rational). $F(z)$ is said to be prime (pseudoprime) if every factorization of the above form implies that $g(z)$ is linear (a polynomial) unless $f(z)$ is linear (rational). If the entire transcendency of $f(z)$ implies the linearity of $g(z)$, then $F(z)$ is said to be right-prime in entire sense.

It is known that a simple geometrical restriction on the distribution of zeros is enough to make zero a Nevanlinna deficient value. In this tendency there have appeared several works [6], [9], [10]. What effects they do have to the factorization theory of meromorphic functions in the above sense is our main problem in this paper. Indeed we shall prove the following

THEOREM 1. Let $F(z)$ be an entre function of hyperorder $\hat{\rho}_{F}$ less than a positive integer $q$. Let $\left\{a_{n}\right\}$ be the set of A-points of $F(z)$. Assume that

and

$$
\sum \frac{1}{\left|a_{n}\right|^{q}}=\infty, \quad \sum \frac{1}{\left|a_{n}\right|^{q+1}}<\infty
$$

$$
\left|\pi-\arg a_{n}\right| \leqq \frac{\pi}{2(q+1)}
$$

Then $F(z)$ is right-prime in entire sense.

Here the hyperorder means

$$
\varlimsup_{r \rightarrow \infty} \frac{\log \log T(r, F)}{\log r} .
$$

This is a precision of Baker's result [1], in which the order finiteness of $F$ is assumed and further the opening of the given sector is assumed to be positive but arbitrarily small. Tsuzuki [11] has given a result of the same kind for $\rho_{F}<1$ (not $\hat{\rho}_{F}$ ) and for the aperture less than $\pi-\delta, \delta>0$. Here $\rho_{F}$ means the order of $F$.

Received Nov. 15, 1974. 


\section{$\S 2$. Lemmas.}

We need several properties on the value distribution.

LEMMA 1. [2]. Let $f(z)$ be an entire function of finite order $\rho_{f} \geqq 1$. Then $f(z)$ takes every value infinitely often with at most one exception in every sector whose aperture is greater than $\pi\left(2-1 / \rho_{f}\right)$.

LEMMA 2. [11]. Let $f(z)$ be an entire function of order less than one and let $\left\{w_{n}\right\}$ be an unbounded sequence for which $f(z)=w_{n}$ has roots only in the sector $|\pi-\arg z| \leqq \omega, 0<\omega<\pi / 2$ for every $n$. Then $f(z)$ is linear.

LEMMA 3. [6]. Let $f(z)$ be an entire function and $g(z)$ the canonical product formed by the zeros of $f(z)$. Assume that the set of zeros $\left\{a_{n}\right\}$ of $f(z)$ satisfies

and

$$
\Sigma\left|a_{n}\right|^{-q}=\infty, \quad \Sigma\left|a_{n}\right|^{-q-1}<\infty
$$

$$
\left|\pi-\arg a_{n}\right| \leqq \frac{\pi}{2(q+1)}
$$

with a positive integer $q$. Then with a positive constant $A(q)$,

$$
\delta(0, f) \geqq \frac{A(q)}{1+A(q)} .
$$

We should remark that in [6] the formulation is less general than the above one and is concerned with only $g$. However the author of [6] knows apparently the above general formulation. For completeness we shall give here a proof of Lemma 3 by making use of several properties in [6].

Proof of Lemma 3. In [6] the following was proved:

$$
\lim _{r \rightarrow \infty} \frac{r^{q}}{m(r, g)}=0 \text {. }
$$

Further it is known that, if $g(z)$ is of order $q+1, \Sigma\left|a_{n}\right|^{-q-1}<\infty$ implies $m(r, g)$ $=o\left(r^{q+1}\right)$. Evidently, if $g(z)$ is of order less than $q+1, m(r, g)=o\left(r^{q+1}\right)$. Let us denote $f(z)$ as $g(z) \exp P(z)$ with transcendental entire or a non-constant polynomial $P(z)$ (the constant case is trivial). Then firstly assuming $p \leqq q$ we have

$$
m(r, g)(1-\varepsilon) \leqq m(r, g)-m\left(r, e^{-P}\right) \leqq m(r, f)
$$

if $P$ is a polynomial of degree $p$. Hence

$$
\begin{aligned}
1-\delta(0, f) & \leqq \varlimsup_{r \rightarrow \infty} \frac{N(r, 0, g)}{m(r, g)} \varlimsup_{r \rightarrow \infty} \frac{m(r, g)}{m(r, f)} \\
& \leqq 1-\delta(0, g) .
\end{aligned}
$$

Therefore by [6]

$$
\delta(0, f) \geqq \delta(0, g) \geqq \frac{A(q)}{1+A(q)}
$$


If $p \geqq q+1, m(r, f) \geqq m\left(r, e^{-P}\right)-m(r, g) \geqq H r^{q+1}$. If $P(z)$ is transcendental, then by Liouville's theorem and Borel-Carathéodory's theorem

$$
\begin{aligned}
m(r, f) & \geqq \frac{1}{3} \log M\left(\frac{r}{2}, e^{P}\right)-m(r, g) \\
& \geqq H r^{q+1} .
\end{aligned}
$$

Thus

$$
1-\delta(0, f) \leqq \varlimsup_{r \rightarrow \infty} \frac{N(r, 0, g)}{m(r, g)} \varlimsup_{r \rightarrow \infty} \frac{m(r, g)}{m(r, f)}=0 .
$$

Therefore $\delta(0, f) \geqq 1$. This completes the proof of Lemma 3 .

We need some growth lemmas.

LEMma 4. [8]. Let $f(z)$ be $\exp L(z)$ with a non-constant $L(z)$. Then the lower order $\mu_{f}$ of $f$ satisfies $\mu_{f} \geqq 1$.

LEMMA 5. [8]. Let $F(z)$ be expressed as $f(g(z))$ with entire $f$ and $g$. If $\mu_{f}>0, \hat{\rho}_{F} \geqq \rho_{g}$.

\section{§3. Proof of Theorem 1 .}

Suppose that $F(z)=f(g(z))$ with transcendental entire $f$ and $g$. Assume that $f(w)=A$ has only finitely many roots. In this case $f(w)=A+P(w) \exp L(w)$ with a polynomial $P$ and entire $L \neq$ const. Then $\mu_{f} \geqq 1$ and hence $\hat{\rho}_{F} \geqq \rho_{g}$. By $\hat{\rho}_{F}<q$ we have $\rho_{g}<q$. Let $p$ be the degree of $P$ and $\left\{w_{j}\right\}$ the set of zeros of $P$. Then

$$
N(r, A, F)=\sum_{\jmath=1}^{p} N\left(r, w_{\jmath}, g\right) \leqq p m(r, g) .
$$

By the assumption on $\left\{a_{n}\right\}$ the order of $N(r, A, F) \geqq q$ and hence $\rho_{g} \geqq q$. This is impossible. Therefore $f(w)=A$ has infinitely many roots $\left\{w_{n}\right\}$. Let $\left\{z_{n l}\right\}$ be the set of $w_{n}$-points of $g(z)$. This is a subset of $\left\{a_{n}\right\}$. Hence for every $n$

$$
\sum_{l=1}^{\infty}\left|z_{n l}\right|^{-q-1}<\infty, \quad\left|\pi-\arg z_{n l}\right| \leqq \frac{\pi}{2(q+1)} .
$$

Assume that there is an index $n$ for which

$$
\sum_{m=1}^{\infty}\left|z_{n m}\right|^{-q}=\infty \text {. }
$$

Then the Picard-Borel theorem implies that

$$
\sum_{m=1}^{\infty}\left|z_{\jmath m}\right|^{-q}=\infty, \quad \jmath=2,3, \cdots
$$

with at most one exception of index, say 1. By Lemma 3

$$
\sum_{\jmath=2}^{\infty} \delta\left(w_{\jmath}, g\right) \geqq \sum_{\jmath=2}^{\infty} \frac{A(q)}{1+A(q)}=\infty,
$$

which is impossible. Hence for any $n$ 


$$
\sum_{m=1}^{\infty} \frac{1}{\left|z_{n m}\right|^{q}}<\infty \text {. }
$$

In this case $\rho_{g} \leqq q$. Assume that $\rho_{g} \geqq 1$. Lemma 1 implies that $g$ attains all the values up to one exception infinitely often in the sector $|\arg z|<\pi-\pi / 2(q+1)$, which is just the complementary sector of the given sector $|\pi-\arg z| \leqq \pi / 2(q+1)$. However all the roots of $g(z)=w_{\jmath}$, that is, $z_{\jmath m}$ lie in the given sector $|\pi-\arg z|$ $\leqq \pi / 2(q+1)$. This is impossible. Assume that $\rho_{g}<1$. Then Lemma 2 gives the linearity of $g(z)$. Thus we have the pseudo-primeness of $F$ in entire sense.

Suppose that $f$ is transcendental entire and $g$ is a polynomial. Then the set of $A$-points of $F$ should be distributed asymptotically as $p$ roots of $A_{p} z^{p}=w_{n}$ for $n \geqq n_{0}$ with $p=\operatorname{deg} g$. If $p \geqq 2$, there appear infinitely many $A$-points of $F$ lying outside the given sector. This is impossible. This gives the right-primeness of $F$ in entire sense.

q. e. d.

We cannot omit the condition $\hat{\rho}_{F}<q$. This is shown by $F(z)=g(z) \exp g(z)$, where $g(z)$ is the canonical product formed by the zeros of $F(z)$. When $\rho_{F}$ (not $\left.\hat{\rho}_{F}\right)$ satisfies $\rho_{F}<q+1$, we can prove our theorem without appealing to Lemma 3. The proof of Corollary 1 indicates this fact.

\section{§4. Corollaries.}

COROLlary 1. Let $F(z)$ be an entire function of finite order $\rho_{F}$. Assume that all the zeros of $F$ lie in the sector $S:|\pi-\arg z| \leqq \pi / 2\left(\rho_{F}+\varepsilon\right), \varepsilon>0$ and that $\rho_{F} \geqq 1$. If $F$ has infinitely many zeros, then $F$ is right-prime in entire sense. If $F$ has only finitely many zeros, then $F$ is pseudo-prime in entire sense.

Proof. The case that $F$ has only finitely many zeros was already proved in [7]. Assume that $F$ has infinitely many zeros. Suppose that $F(z)=f(g(z))$ with transcendental entire $f$ and $g$. Then $\rho_{f}=0, \rho_{g} \leqq \rho_{F}$. Let $\left\{w_{n}\right\}$ and $\left\{z_{n l}\right\}$ be the set of zeros of $f$ and the roots of $g(z)=w_{n}$ respectively. $\left\{z_{n l}\right\}$ is an infinite set except for at most one index of $n$, say 1 . $\left\{z_{n l}\right\} \in S$. If $\rho \geqq 1$, this is impossible by Lemma 1 . If $\rho_{g}<1$, Lemma 2 gives us a contradiction. The rightprimeness in entire sense is almost trivial.

q.e. d.

COROLlARY 2. Let $F(z)$ be an entire function of hyperorder less than a positive integer $q$. Assume that the set $\left\{a_{n}\right\}$ of zeros of $F^{\prime}(z)$ satisfies the conditions in Theorem 1. Then $F(z)$ is right-prime in entrre sense.

Proof. Consider the derived equation $F^{\prime}(z)=f^{\prime}(g(z)) g^{\prime}(z) . \quad \hat{\rho}_{F}=\hat{\rho}_{F^{\prime}}$ is almost trivial. If $f^{\prime}(w)=0$ has only finitely many roots, $f^{\prime}(w)=P(w) \exp L(w)$ with a polynomial $P(w)$ and entire $L(w) \neq$ const. Then $\mu_{f^{\prime}}=\mu_{f} \geqq 1$. Therefore $\hat{\rho}_{F}=$ $\hat{\rho}_{f(g)} \geqq \rho_{g^{\prime}}$ which shows $\rho_{g}<q$. Let $p$ be the degree of $P(w)$. Then

$$
\begin{aligned}
N\left(r, 0, F^{\prime}\right) & =\sum_{j=1}^{p} N\left(r, w_{\jmath}, g\right)+N\left(r, 0, g^{\prime}\right) \\
& \leqq(p+1)(1+\varepsilon) m(r, g) .
\end{aligned}
$$


Since the order of $N\left(r, 0, F^{\prime}\right)$ is not less than $q$, we have $\rho_{g} \geqq q$. This is impossible. Hence $f^{\prime}(w)$ has infinitely many zeros. The remaining part can be proved quite similarly as in Theorem 1.

q. e. d.

COROLlaRy 3. Under the same assumptions as in Corollary 1, $F$ is prime, if $F$ has infinitely many zeros including at least one simple zero.

Proof. $F(z)$ is right-prime in entire sense by Corollary 1. Suppose that

$$
F(z)=A\left\{g(z)-w_{1}\right\}^{m_{i}} \ldots\left\{g(z)-w_{k}\right\}^{m_{k}} .
$$

Evidently $\rho_{g}=\rho_{F} \geqq 1$. Assume that $g(z)=w_{1}$ and $g(z)=w_{2}$ have infinitely many roots. Then Lemma 1 implies a contradiction. Assume that $g(z)=w_{1}$ has only finitely many roots. Then

$$
g(z)=w_{1}+P(z) e^{Q(z)},
$$

where $P, Q$ are polynomials and the degree of $Q=q$. Hence $\rho_{g}=q$. In this case $P e^{\ell}=w_{2}-w_{1}$ has roots whose arguments have $2 q$ asymptotic directions $d_{1}, \cdots, d_{2 q}$. They satisfy

$$
d_{\jmath}-d_{\jmath-1}=\frac{2 \pi}{2 q}, \quad \jmath=1, \cdots, 2 q, \quad d_{0}=d_{2 q}-2 \pi .
$$

Hence $g(z)=w_{2}$ has roots lying outside $|\pi-\arg x| \leqq \pi / 2\left(\rho_{g}+\varepsilon\right)$, which is again impossible. Hence $k$ should be equal to 1 . By the existence of a simple zero of $F(z)$ we have $F(z)=A\left(g(z)-w_{1}\right)$. This means that $F(z)$ is prime in entire sense. Evidently $F$ is not periodic. Then by Gross' theorem [3], $F$ is prime.

q. e. d.

This corollary gives an extension of Kimura's theorems in [4], [5]. Indeed he assumed that all the zeros are negative and that $n(r, 0)$ satisfies certain regularity conditions such as $n(r, 0) \sim a r^{\rho}$ or so. His proof in both theorems depended heavily upon certain Tauberian theorems.

\section{BIBLIOGRAPHY}

[1] BAKER, I.N., The value distribution of composite entire functions, Acta Sci. Math. (Szeged) 32 (1971), 87-90.

[2] Bieberbach, L., Über eine Vertiefung des Picardschen Satzes bei ganzen Funkt1onen endlicher Ordnung, Math. Zeits., 3 (1919), 175-190.

[3] Gross, F., Factorization of entire functions which are periodic mod $g$, Indian Journ. pure Appl. Math., 2 (1971), 561-571.

[4] Kimura, S., On prime entire functıons, Kōdai Math. Sem. Rep., 24 (1972), $28-33$.

[5] Kimura, S., On prime entıre functions, II. Kōdai Math. Sem. Rep., 24 (1972), 277-280.

[6] Kовауаshi, T., On the deficiency of an entire function of finite genus, Kōdai Math. Sem. Rep., 27 (1976), 320-328. 
[7] Ozawa, M., On the solution of functional equation $f \circ g(z)=F(z)$, Kōdai Math. Sem. Rep., 20 (1968), 159-162.

[8] OzAwa, M., On the solution of functional equation $f \circ g(z)=F(z)$, III. Kōdai Math. Sem. Rep., 20 (1968), 257-263.

[9] OzAwA, M., Radial distribution of zeros and deficiency of a canonical product of finite genus, Kōdai Math. Sem. Rep., 25 (1973), 502-512.

[10] Ozawa, M., Distribution of zeros and deficiency of a canonical product of genus one, Hokkaido Math. Journ., 3 (1974), 218-231.

[11] Tsuzuki, M., On the value distribution of entire functions of order less than one. Journ. Saitama Univ., 10 (1974), 1-4.

Department of Mathematics

Tokyo Institute of Technology

Oh-okayama, Meguro-Ku, Tokyo

JAPAN 